\title{
COMPLETENESS, METRIZABILITY AND COMPACTNESS IN SPACES OF FUZZY-NUMBER-VALUED FUNCTIONS
}

\author{
J.J. FONT, D. SANCHIS, AND M. SANCHIS
}

\begin{abstract}
Fuzzy-number-valued functions, that is, functions defined on a topological space taking values in the space of fuzzy numbers, play a central role in the development of Fuzzy Analysis. In this paper we study completeness, metrizability and compactness of spaces of continuous fuzzy-number-valued functions.
\end{abstract}

\section{INTRODUCTION}

Fuzzy Analysis is based on the notion of fuzzy number in the same way as Classical Analysis is based on the concept of real number. From 1986, the so-called representation theorem of real fuzzy numbers (see [19]) eased considerably the development of the theory concerning fuzzy-number-valued functions, that is, functions defined on a topological space taking values in $\mathbb{E}^{1}$, the space of fuzzy numbers. Such functions, as real-valued functions do in the classical setting, play a central role in Fuzzy Analysis. Namely, fuzzy-number-valued functions have become the main tool in several fuzzy contexts, such as fuzzy differential equations ([6]), fuzzy integrals $([37],[41])$, fixed point theory $([25,30,40])$ and fuzzy optimization $([20],[38],[39])$.

In this paper we address three topological aspects of the spaces of continuous fuzzy-number-valued functions when endowed with the most usual topologies. Namely we will study completeness, metrizability and compactness in this context. Only the latter concept, which is clearly related to Ascoli theorem, seems to have received certain attention in fuzzy literature (see, e.g., [33], [14]). The prototype of such result in Classical Analysis was proved by Ascoli in [5] and, independently, by Arzelà, who acknowledged Ascoli's priority in [4]. Nowadays, Arzelà-Ascoli type theorems encompass the study of the (relative) compacity of a family of functions endowed with several topologies and their literature is extense. The applications of these results are numerous in different settings; namely, in the context of differential equations, in finding extremal curves, in most criteria for the consistency of systems involving inequalities, etc.

In [14, Theorem 4.2], Fang and Xue set up a fuzzy version of Ascoli theorem which characterized compact subsets of the space $C\left(K,\left(\mathbb{E}^{1}, d_{\infty}\right)\right)$ of all fuzzy-valued continuous functions on a compact metric space $K$ endowed with the topology of the uniform convergence. Unfortunately this version is not correct since, as pointed out in [15], it is based on a wrong characterization ([14, Theorem 2.4]) of the compact subsets of $\left(\mathbb{E}^{1}, d_{\infty}\right)$. In the last section of this paper we fix [14, Theorem 4.2] by

Key words and phrases. Continuous fuzzy number-valued functions; Metrizability; Completeness; bounded sets; Ascoli theorem.

This research is supported by Spanish Government (grant MTM2016-77143-P). 
extending the fuzzy Ascoli theorem to a broader framework. The key concepts in our approach are bounded subsets of a topological space and $\alpha_{f}$-spaces. Thus, previously, in Section 3 we obtain a fuzzy characterization of bounded subsets and $\alpha_{f}$-spaces, and point out how such spaces appear in a natural way when considering the completeness of the spaces $C\left(X,\left(\mathbb{E}^{1}, d_{\infty}\right)\right), X$ a topological space, equipped with the topology $\tau_{\alpha}$ of uniform convergence on the (bounded) members of a cover $\alpha$ of $X$. In Section 4, we establish an explicit criterion for $C_{\tau_{\alpha}}\left(X,\left(\mathbb{E}^{1}, d_{\infty}\right)\right)$ to be metrizable. Finally, as mentioned above, in Section 5 we address the fuzzy Ascoli theorem (and also the weak fuzzy Ascoli theorem) for spaces $C\left(X,\left(\mathbb{E}^{1}, d_{\infty}\right)\right)$. As a consequence of our results we show that $C\left(X,\left(\mathbb{E}^{1}, d_{\infty}\right)\right)$ endowed with the topology of the uniform convergence satisfies the fuzzy Ascoli theorem if and only if $X$ is pseudocompact.

\section{Preliminaries AND NOtation}

Given a fuzzy subset $u$ on the real numbers $\mathbb{R}$, the $\lambda$-level set of $u$ is defined by $[u]^{\lambda}=\{x \in \mathbb{R}: u(x) \geq \lambda\}$ for $\lambda \in(0,1]$ and $[u]^{0}=\operatorname{cl}_{\mathbb{R}}\{x \in \mathbb{R}: u(x)>0\}$ for $\lambda=0$.

Now, the fuzzy number space $\mathbb{E}^{1}$ is the set of such $u$ satisfying the following properties:

(1) $u$ is normal, i.e., there exists an $x_{0} \in \mathbb{R}$ with $u\left(x_{0}\right)=1$.

(2) $u$ is convex, i.e., $u(\lambda x+(1-\lambda) y) \geq \min \{u(x), u(y)\}$ for all $x, y \in \mathbb{R}, \lambda \in[0,1]$.

(3) $u(x)$ is upper-semicontinuous.

(4) $[u]^{0}$ is a compact set in $\mathbb{R}$.

Notice that, if $u \in \mathbb{E}^{1}$, then the $\lambda$-level set $[u]^{\lambda}$ of $u$ is a compact interval for each $\lambda \in[0,1]$. We denote $[u]^{\lambda}$ by $\left[u^{-}(\lambda), u^{+}(\lambda)\right]$. Every real number $r$ can be consider a fuzzy number: indeed, if $r$ can be identified with the fuzzy number $\tilde{r}$ defined by

$$
\tilde{r}= \begin{cases}1 & \text { if } t=r \\ 0 & \text { if } t \neq r .\end{cases}
$$

From now on, we do not distinguish between $r$ and $\tilde{r}$. The following two results are useful in the theory of fuzzy numbers.

THEOREM 2.1. [19] Let $u \in \mathbb{E}^{1}$ and $[u]^{\lambda}=\left[u^{-}(\lambda), u^{+}(\lambda)\right], \lambda \in[0,1]$. Then the pair of functions $u^{-}(\lambda)$ and $u^{+}(\lambda)$ has the following properties:

(i) $u^{-}(\lambda)$ is a bounded left continuous nondecreasing function on $(0,1]$.

(ii) $u^{+}(\lambda)$ is a bounded left continuous nonincreasing function on $(0,1]$.

(iii) $u^{-}(\lambda)$ and $u^{+}(\lambda)$ are right continuous at $\lambda=0$.

(iv) $u^{-}(1) \leq u^{+}(1)$.

Conversely, if the pair of functions $\alpha(\lambda)$ and $\beta(\lambda)$ satisfies the above conditions (i)-(iv), then there exists a unique $u \in \mathbb{E}^{1}$ such that $[u]^{\lambda}=[\alpha(\lambda), \beta(\lambda)]$ for each $\lambda \in[0,1]$.

THEOREM 2.2. [19, 12] For $u, v \in \mathbb{E}^{1}$, define

$$
d_{\infty}(u, v)=\sup _{\lambda \in[0,1]} \max \left\{\left|u^{-}(\lambda)-v^{-}(\lambda)\right|,\left|u^{+}(\lambda)-v^{+}(\lambda)\right|\right\} .
$$


Then $d_{\infty}$ is a metric on $\mathbb{E}^{1}$ called the supremum metric on $\mathbb{E}^{1}$, and $\left(\mathbb{E}^{1}, d_{\infty}\right)$ is a complete metric space.

Notice that, by the definition of $d_{\infty}$, the reals $\mathbb{R}$ endowed with the euclidean topology can be topologically identified with the closed subspace $\tilde{R}=\{\tilde{x}: x \in \mathbb{R}\}$ of $\left(\mathbb{E}^{1}, d_{\infty}\right)$ where $\tilde{x}^{+}(\lambda)=\tilde{x}^{-}(\lambda)=x$ for all $\lambda \in[0,1]$. As a metric space, we will always consider $\mathbb{E}^{1}$ equipped with the metric $d_{\infty}$.

Throughout this paper, $X$ will stand for a Tychonoff space, that is, a completely regular Hausdorff space. A subset $B$ of a space $X$ is said to be bounded (in $X$ ) if every real-valued continuous function on $X$ is bounded on $B$ or, equivalently, every locally finite sequence $\left\{U_{n}: n \in \mathbb{B}\right\}$ of pairwise disjoint open sets meeting $B$ is finite. Spaces which are bounded in themselves are called pseudocompact. Given a space $X$, the family of all bounded subsets of $X$ is denoted by $\beta$. If $\alpha$ is a cover of a space $X$, we say that a function $f$ from a space $X$ into a space $Y$ is $\alpha_{f}$-continuous if the restriction of $f$ to each member of $\alpha$ can be extended to a continuous function on the whole $X$. A space $X$ is called an $\alpha_{f}$-space if every real-valued $\alpha_{f}$-continuous function on $X$ is continuous.

For $\alpha \subseteq \beta$, locally pseudocompact spaces and $k_{r}$-spaces (spaces $X$ where a realvalued function is continuous whenever its restriction to each compact subset of $X$ is continuous) are examples of $\alpha_{f}$-spaces. Thus, locally compact spaces, first countable spaces (in particular, metrizable spaces) are $\alpha_{f}$-spaces too. The theory of $z$-closed projections [27], the distribution of the functor of the Dieudonné completion [9, 31], compactness of functions spaces in the topology of the pointwise convergence [2], and locally pseudocompact groups [34] are some of the frameworks where $\alpha_{f}$-spaces arise in a natural way. We encourage the reader unfamiliar with the techniques of the theory of bounded subsets to consult [35].

Let $F\left(X, \mathbb{E}^{1}\right)$ denote the set of all functions from a set $X$ into $\mathbb{E}^{1}$. For a cover, say $\alpha$, of $X$, we denote by $\tau_{\alpha}$ the topology of uniform convergence on members of $\alpha$. It is a well-known fact that $\left(\left(F\left(X, \mathbb{E}^{1}\right), \tau_{\alpha}\right)\right.$ is a Tychonoff space. Indeed, the family of all subsets of $F\left(X, \mathbb{E}^{1}\right) \times F\left(X, \mathbb{E}^{1}\right)$ of the form

$$
U(A, \varepsilon)=\left\{(f, g) \in F\left(X, \mathbb{E}^{1}\right) \times\left(F\left(X, \mathbb{E}^{1}\right): \sup _{a \in A} d_{\infty}(f(a), g(a))<\varepsilon\right\},\right.
$$

for all $A \in \alpha$ and all $\varepsilon>0$, is a subbase for a (Hausdorff) uniformity $\mathscr{U}_{\alpha}$ on $F\left(X, \mathbb{E}^{1}\right)$ which induces the topology $\tau_{\alpha}$. In the sequel we will use the well-known fact that every uniformity with a countable base is metrizable (see, e.g., [13, Theorem 8.1.21]).

Our terminology and notation are standard. For instance, $\mathbb{N}$ stands for the set of natural numbers and $\left.f\right|_{A}$ means the restriction of a function $f$ to a subset $A$. For a subset $\mathscr{F}\left(X, \mathbb{E}^{1}\right)$ of $F\left(X, \mathbb{E}^{1}\right)$, the space $\left(\mathscr{F}\left(X, \mathbb{E}^{1}\right), \tau_{\alpha}\right)$ is denoted by $\mathscr{F}_{\tau_{\alpha}}\left(X, \mathbb{E}^{1}\right)$. The symbol $C\left(X, \mathbb{E}^{1}\right)$ (respectively, $C(X, \mathbb{R})$ ) stands for the set of all continuous functions from $X$ into $\left(\mathbb{E}^{1}, d_{\infty}\right)$, that is, the set of all fuzzy-number-valued continuous functions on $X$ (respectively, the set of all real-valued continuous functions on $X$ ). Notice that we obtain $\tau_{p}$, the topology of the pointwise convergence, by taking $\alpha$ the cover of $X$ consisting of its points (equivalently, of all its finite subsets). The paper 
[23] covers a wide variety of topics about $C\left(X, \mathbb{E}^{1}\right)$ endowed with the topology $\tau_{p}$. If $\alpha=\{X\}$, then we obtain the topology, $\tau_{u}$, of uniform convergence on $X$. The cover $k$ of all compact subsets of a topological space $X$ induces the so-called compactopen topology on $C\left(X, \mathbb{E}^{1}\right)$ denoted by $\tau_{c o}$. It is worth noting that the pointwise convergence topology on $F\left(X, \mathbb{E}^{1}\right)$ coincides with the product topology on $\left(\mathbb{E}^{1}\right)^{X}$. This equivalent to consider $\tau_{p}$ on $C\left(X, \mathbb{E}^{1}\right)$ when $X$ is equipped with the discrete topology. For notions which are not explicitly defined here, the reader might consult [13].

\section{Completeness of $C_{\tau_{\alpha}}\left(X, \mathbb{E}^{1}\right)$}

In the first part of this section we shall show that the notions of bounded subset and of $\alpha_{f}$-space $(\alpha \subseteq \beta$ ) can be characterized by means of fuzzy-number-valued continuous functions. In the second part we shall show that $\alpha_{f}$-spaces occur naturally when we study completeness of $C_{\tau_{\alpha}}\left(X, \mathbb{E}^{1}\right)$. A similar claim can be made about the metrizability of $C_{\tau_{\alpha}}\left(X, \mathbb{E}^{1}\right)$ as we will see in the next section.

A subset $A$ of a metric space $(X, d)$ is precompact if for every $\varepsilon>0$, there is a finite subset $\left\{x_{1}, x_{2}, \ldots, x_{n}\right\}$ such that $A \subseteq \bigcup_{i=1}^{n} B_{\varepsilon}\left(x_{i}\right)$ where, as usual, $B_{\varepsilon}\left(x_{i}\right)$ stands for the ball of center $x_{i}$ and radius $\varepsilon$. Recall that a metric space $X$ is compact if and only if $X$ is precompact and complete. Since boundedness is equivalent to precompactness for subsets of $\mathbb{R}$, we can replace bounded by precompact in the definition of bounded subset. In this direction, we have

THEOREM 3.1. For a subset $B$ of a space $X$, the following are equivalent:

(1) $B$ is bounded in $X$.

(2) For each continuous function $f: X \rightarrow \mathbb{E}^{1}, f(B)$ is precompact.

Proof. $(2) \Longrightarrow(1)$ follows from the fact that precompact subsets of $\mathbb{R}$ are precompact in $\mathbb{E}^{1}$. In order to prove $(1) \Longrightarrow(2)$, notice that, being $\mathbb{E}^{1}$ a complete metric space of non-measurable cardinality, it is realcompact. Thus, by $\left[18\right.$, Theorem 11.8], $\mathbb{E}^{1}$ is homeomorphic to a closed subset $T$ of a product, $\mathbb{R}^{S}$, of real lines. Now, if $f: X \rightarrow \mathbb{E}^{1}$ is a continuous function, we can consider the bounded subset $\left(\pi_{s} \circ\right.$ $f)(B)$ for all $s \in S$. Then $\operatorname{cl}_{\mathbb{E}^{1}} f(B)=\operatorname{cl}_{T} f(B)$ is a closed subset of the compact set $\left.\prod_{s \in S} \operatorname{cl}_{\mathbb{R}}\left(\pi_{s} \circ f\right)\right)(B)$ and, consequently, $\mathrm{cl}_{\mathbb{E}^{1}} f(B)$ is compact. Thus, $f(B)$ is precompact.

THEOREM 3.2. If $\alpha \subseteq \beta$, then the following are equivalent:

(1) $X$ is an $\alpha_{f}$-space.

(2) Every $\alpha_{f}$-continuous function from $X$ into $\mathbb{E}^{1}$ is continuous.

Proof. Since $\mathbb{E}^{1}$ is a Tychonoff space, $(1) \Longrightarrow(2)$ is a consequence of Lemma 8 in [7]. Thus, we only need to prove $(2) \Longrightarrow(1)$. To this end, it suffices to notice that if $f: X \rightarrow \mathbb{R}$ is an $\alpha_{f}$-continuous function, then $f$ can be regarded as an $\alpha_{f}$-continuous function from $X$ into $\mathbb{E}^{1}$ because $\mathbb{R}$ is a (closed) subspace of $\mathbb{E}^{1}$.

Let us now move on to the study of completeness of $C_{\tau_{\alpha}}\left(X, \mathbb{E}^{1}\right)$. We say that a space $X$ is topologically complete if $X$ is homeomorphic to a closed subspace of a product of metrizable spaces. It is known that, for every space $X$, there exists a unique, up to homeomorphisms that leave $X$ pointwise fixed, topologically complete 
space $\gamma X$, in which $X$ is dense and every continuous function $f$ from $X$ into a topologically complete space $M$ can be extended to a continuous function $f^{\gamma}$ on $\gamma X$. Such $\gamma X$ is called the Dieudonné topological completion of $X$. For these and related results, the reader might consult [18]. Bounded subsets can be characterized as subsets whose closure in $\gamma X$ is compact (see [18, Problem 8E.] or [3, Proposition 5.1]). The following lemma is straightforward. We write $\tau_{1} \geq \tau_{2}$, if the topology $\tau_{1}$ is finer than the topology $\tau_{2}$.

LEMMA 3.3. Let $X$ be a space. If $\tau \geq \tau_{p}$, then $C_{\tau}(X, \mathbb{R})$ is topologically embedded as a closed subspace of $C_{\tau}\left(X, \mathbb{E}^{1}\right)$.

It is a well-known fact that, for an arbitrary space $X, C_{\tau_{u}}(X,(M, d))$ is complete whenever the metric space $(M, d)$ is (see, for example, [13, Exercise 8.3.C.(a)]). Thus, we have

THEOREM 3.4. (Compare with [14, Theorem 3.5]) For an arbitrary space $X$, $C_{\tau_{u}}\left(X, \mathbb{E}^{1}\right)$ is a complete metric space.

Proof. The space $\left(\mathbb{E}^{1}, d_{\infty}\right)$ being complete [19], completeness follows from [13, Exercise 8.3.C.(a)]. On the other hand, notice that the entourage of the diagonal

$$
\left\{(f, g) \in C\left(X, \mathbb{E}^{1}\right) \times\left(C\left(X, \mathbb{E}^{1}\right): \sup _{x \in X} d_{\infty}(f(x), g(x))<1 / n\right\}\right.
$$

for all $n \in \mathbb{N}$, forms a countable subbase for the uniformity of $C_{\tau_{u}}\left(X, \mathbb{E}^{1}\right)$. Thus, $C_{\tau_{u}}\left(X, \mathbb{E}^{1}\right)$ is metrizable by [13, Theorem 8.1.21].

Now we take up a result which plays a pivotal role in the characterization of the completeness of $C_{\tau_{\alpha}}\left(X, \mathbb{E}^{1}\right)$. Recall that there exists an isometric embedding $j$ of $\left(\mathbb{E}^{1}, d_{\infty}\right)$ into a Banach space $(E,\|\cdot\|)$ which preserves convex combinations, that is, $j$ satisfies the following two properties: $(1) d_{\infty}(u, v)=\|j(u)-j(v)\|$ for all $u, v \in \mathbb{E}^{1}$, and $(2) j(\lambda u+(1-\lambda) v)=\lambda j(u)+(1-\lambda) j(v)$ for all $u, v \in \mathbb{E}^{1}$ and all $\lambda \in[0,1]$ (see [11] for the details). Moreover, $j\left(\mathbb{E}^{1}\right)$ is a closed cone of $(E,\|\cdot\|)$ with vertex 0 . Recall also that a subset $A$ of a space $X$ is called $C$-embedded in $X$ if every real-valued continuous function on $A$ extends continuously to the whole $X$.

THEOREM 3.5. For a subset $A$ of a space $X$, the following assertions are equivalent: (1) $A$ is $C$-embedded in $X$.

(2) Every continuous fuzzy-number-valued function $f$ on $A$ with separable range has a continuous extension $F$ to the whole $X$; furthermore $F(X)$ is included in the closed convex hull of $f(A)$.

Proof. (2) $\Longrightarrow(1)$ can be easily verified since $\mathbb{R}$ is a closed convex subset of $\mathbb{E}^{1}$. In order to see $(1) \Longrightarrow(2)$, let $j$ be the above isometric embedding of $\left(\mathbb{E}^{1}, d_{\infty}\right)$ into a Banach space $(E,\|\cdot\|)$. From now on, we identify $\mathbb{E}^{1}$ with $j\left(\mathbb{E}^{1}\right)$. Thus, we can consider $f$ as a function from $A$ to the closed (so complete) convex hull $\overline{c o n v}(f(A))$ of $f(A)$ in $(E,\|\cdot\|)$. Being $\mathbb{E}^{1}$ a (complete) cone, we have that $\overline{c o n v}(f(A)) \subset$ $\mathbb{E}^{1}$. Since $A$ is $C$-embedded in $X$, by Theorem 2.4 of [17] and Theorem 4.7 of [36], every continuous pseudometric $d$ on $A$ which induces a separable topology extends continuously to a continuous pseudometric on $X$. Hence we can apply 
Theorem 2.3 of [1] to conclude that $f$ has a continuous extension $F$ to $X$ with $F(X) \subset \overline{\operatorname{conv}}(f(A))$.

REMARK 3.6. An argument similar to the one used in the previous theorem enables us to characterize $C^{\star}$-embedded subsets $A$ (that is, every bounded real-valued continuous function on $A$ extends to a continuous function on $X$ ). Indeed, we can apply Theorem 2.9 of [1] to obtain: For a subset $A$ of a space $X$, the following assertions are equivalent: (1) $A$ is $C^{\star}$-embedded in $X$, and (2) every continuous fuzzy-numbervalued function $f$ on $A$ with precompact range has a continuous extension $F$ to the whole $X$; furthermore $F(X)$ is included in the closed convex hull of $f(A)$.

The relationship between $\alpha_{f}$-spaces and the completeness of $C_{\tau_{\alpha}}\left(X, \mathbb{E}^{1}\right)$ is given by the next result. Let us first recall that, given a space $X$, a family $\alpha \subseteq \beta$ is a bornology if it satisfies the following two conditions:

(i) $\alpha$ is a cover of $X$; and

(ii) if $A$ and $B$ belong to $\alpha$, then there exists $C \in \alpha$ such that both $A$ and $B$ are included in $C$.

Indeed, any cover $\alpha$ of $X$ generetes a bornology $\widehat{\alpha}$ by taking finite unions of elements of $\alpha$ and both uniformities coincide.

THEOREM 3.7. Assume $\alpha \subseteq \beta$ is a bornology in a space $X$. Then $C_{\tau_{\alpha}}\left(X, \mathbb{E}^{1}\right)$ is complete if and only if $X$ is an $\alpha_{f}$-space.

Proof. Assume that $X$ is an $\alpha_{f^{-}}$space and consider a Cauchy net $\left\{f_{i}\right\}_{i \in I}$ in $C_{\tau_{\alpha}}\left(X, \mathbb{E}^{1}\right)$. By [19], $\left(\mathbb{E}^{1}, d_{\infty}\right)$ is a complete metric space and consequently each element $f_{i}$ has a continuous extension, say $f_{i}^{\gamma}$, to $\gamma X$. By Theorem 3.4, for each subset $B$ in $\alpha$, the net $\left\{\left.f_{i}^{\gamma}\right|_{\mathrm{cl}_{\gamma X} B}\right\}_{i \in I}$ converges uniformly to a function $f_{B} \in C\left(\mathrm{cl}_{\gamma X} B, \mathbb{E}^{1}\right)$. Let $g$ be the function from $X$ into $\left(\mathbb{E}^{1}, d_{\infty}\right)$ defined by the rule $\left.g\right|_{B}=f_{B}$ whenever $B$ is a bounded subset of $X$ in $\alpha$. Since $\alpha$ is a bornology, a standard argument shows that $g$ is well defined. Moreover, since each compact subset of a topological space is $C$-embedded, by Theorem 3.5 the restriction of $g$ to each subset $B \in \alpha$ can be continuously extended to $\gamma X$. Thus, $g$ is $\alpha_{f}$-continuous. Since $X$ is an $\alpha_{f}$-space, $g$ is actually continuous. One can easily verify that $g$ is the limit of the net $\left\{f_{i}\right\}_{i \in I}$ in $C_{\tau_{\alpha}}\left(X, \mathbb{E}^{1}\right)$. Thus, the function space $C_{\tau_{\alpha}}\left(X, \mathbb{E}^{1}\right)$ is complete.

Suppose now that $C_{\tau_{\alpha}}\left(X, \mathbb{E}^{1}\right)$ is complete. Let $f$ be a $\alpha_{f}$-continuous fuzzy-numbervalued function on $X$ and consider the set $\{B \subset X: B \in \alpha\}$ directed by inclusion. Define now a net $\left\{f_{B}\right\}_{B \in \alpha}$ where $f_{B}$ is the continuous extension of $\left.f\right|_{B}$ to $X$. It is clear that the net $\left\{f_{B}\right\}_{B \in \alpha}$ converges to $f$ in $F_{\tau_{\alpha}}\left(X, \mathbb{E}^{1}\right)$. The completeness of $C_{\tau_{\alpha}}\left(X, \mathbb{E}^{1}\right)$ implies that $f$ is a continuous function. Thus, $X$ is an $\alpha_{f}$-space.

COROLLARY 3.8. (1) $C_{\tau_{\beta}}\left(X, \mathbb{E}^{1}\right)$ is complete if and only if $X$ is a $b_{f}$-space.

(2) $C_{\tau_{c o}}\left(X, \mathbb{E}^{1}\right)$ is complete if and only if $X$ is a $k_{r}$-space.

(3) $C_{\tau_{p}}\left(X, \mathbb{E}^{1}\right)$ is complete if and only if $X$ is discrete.

\section{Metrizability of $C_{\tau_{\alpha}}\left(X, \mathbb{E}^{1}\right)$}

We now address the question of metrizability of the space $C_{\tau_{\alpha}}\left(X, \mathbb{E}^{1}\right)$. The following is an explicit criterion for $C_{\tau_{\alpha}}\left(X, \mathbb{E}^{1}\right)$ to be metrizable. Let $\alpha \subseteq \beta$ be a 
cover of a space $X$. We say that $X$ is hemi- $\alpha$-bounded if there is a countable family $\mathscr{A}=\left\{A_{n}: n \in \mathbb{N}\right\} \subset \alpha$ such that $X=\bigcup_{n \in \mathbb{N}} A_{n}$, and any $A \in \alpha$ is a subset of some finite union $A_{n_{1}} \cup A_{n_{2}} \cup \cdots A_{n_{k}}$ of elements of $\mathscr{A}$. Hemi- $\beta$-bounded (respectively, hemi- $k$-bounded) spaces are usually called hemibounded (respectively, hemicompact) spaces.

THEOREM 4.1. Let $\alpha$ be a cover of a space $X$. If every element of $\alpha$ is a closed set, then the following conditions are equivalent:

(1) $X$ is a hemi- $\alpha$-bounded space.

(2) $C_{\tau_{\alpha}}\left(X, \mathbb{E}^{1}\right)$ is metrizable.

(3) $C_{\tau_{\alpha}}\left(X, \mathbb{E}^{1}\right)$ is first countable.

(4) $C_{\tau_{\alpha}}\left(X, \mathbb{E}^{1}\right)$ has a countable base at the zero function.

Proof. (1) $\Longrightarrow(2)$ Let $\left\{A_{n}: n \in \mathbb{N}\right\} \subset \alpha$ be a countably family which witnesses hemi$\alpha$-boundedness of $X$. It is an easy matter to check that

$$
\mathscr{B}=\left\{U\left(A_{n}, 1 / k\right): n, k \in \mathbb{N}\right\}
$$

is a countable subbase for the uniformity $\mathscr{U}_{\alpha}$. The result now follows from $[13$, Theorem 8.1.21].

$(2) \Longrightarrow(3)$ and $(3) \Longrightarrow(4)$ are trivial. To see $(4) \Longrightarrow(1)$, set

$$
V(A, \varepsilon):=\left\{f \in C\left(X, \mathbb{E}^{1}\right): \sup _{x \in A} d_{\infty}(0, f(x))<\varepsilon\right\}
$$

with $A \in \alpha$ and $\varepsilon>0$, and consider a countable family $\mathscr{B}=\left\{\left(V\left(A_{n}, \varepsilon_{n}\right): n \in \mathbb{N}\right\}\right.$ such that finite intersections of elements of $\mathscr{B}$ form a base at 0 .

Fix now $A \in \alpha$. Given a neighborhood $V(A, 1 / 2)$ of 0 , there exist

$$
\left\{V\left(A_{n_{1}}, \varepsilon_{n_{1}}\right), V\left(A_{n_{2}}, \varepsilon_{n_{2}}\right), \ldots, V\left(A_{n_{k}}, \varepsilon_{n_{k}}\right)\right\}
$$

such that

$$
V\left(A_{n_{1}}, \varepsilon_{n_{1}}\right) \cap V\left(A_{n_{2}}, \varepsilon_{n_{2}}\right) \cap \ldots \cap V\left(A_{n_{k}}\right) \subset V(A, 1 / 2) .
$$

We claim that $A \subseteq A_{n_{1}} \cup A_{n_{2}} \cup \cdots \cup A_{n_{k}}$. Indeed, suppose, contrary to what we claim, that there is $x \in A \backslash\left(A_{n_{1}} \cup A_{n_{2}} \cup \cdots \cup A_{n_{k}}\right)$. The set $A_{n_{1}} \cup A_{n_{2}} \cup A_{n_{k}}$ being closed, there is $f \in C\left(X, \mathbb{E}^{1}\right)$ such that $f(x)=1$ and $\left.f\right|_{A_{n_{1}} \cup A_{n_{2}} \cup A_{n_{k}}}=0$ (see Proposition 3.5 of [23]). Then $f \in V\left(A_{n_{1}}, \varepsilon_{n_{1}}\right) \cap V\left(A_{n_{2}}, \varepsilon_{n_{2}}\right) \cap \ldots \cap V\left(A_{n_{k}}\right)$ but $f \notin V(A, 1 / 2)$. This contradiction yields the claim.

In addition, for any $x \in X$, there exists $A \in \alpha$ containing $x$. Hence the previous argument proves that there are $A_{n_{1}}, A_{n_{2}}, \cdots, A_{n_{k}}$ with $A \subset A_{n_{1}} \cup A_{n_{2}} \cup \cdots \cup A_{n_{k}}$. Thus, the family $\left\{A_{n}: n \in \mathbb{N}\right\}$ is a cover of $X$. We have just showed that $\left\{A_{n}: n \in \mathbb{N}\right\}$ witnesses the hemi- $\alpha$-boundedness of $X$.

REMARK 4.2. (1) The condition that the elements of the cover $\alpha$ are closed is not as restrictive as it may seem. Indeed, if $\alpha \subseteq \beta$ is a cover of $X$, then we can consider the cover $\widehat{\alpha} \subseteq \beta$ defined as

$$
\widehat{\alpha}=\left\{\operatorname{cl}_{X} A: A \in \alpha\right\} .
$$

Then the function spaces $C_{\tau_{\alpha}}\left(X, \mathbb{E}^{1}\right)$ and $C_{\tau_{\widehat{\alpha}}}\left(X, \mathbb{E}^{1}\right)$ are homeomorphic. An even more strong conclusion is possible: actually, they are uniformly isomorphic. The proof can be left to the reader as an easy exercise. 
(2) For the function space $C_{\tau_{\alpha}}(X, \mathbb{R})$, the equivalence of (2) and (4) is a consequence of the well-known Birkhoff-Kakutani theorem which states that a topological group $G$ is metrizable if and only if it is Hausdorff and first countable. However, it is worth noting that $C_{\tau_{\alpha}}\left(X, \mathbb{E}^{1}\right)$ fails to be a topological group so that Birkhoff-Kakutani theorem does not apply in this context.

COROLLARY 4.3. (1) $C_{\tau_{\beta}}\left(X, \mathbb{E}^{1}\right)$ is metrizable if and only if $X$ is hemibounded.

(2) $C_{\tau_{c o}}\left(X, \mathbb{E}^{1}\right)$ is metrizable if and only if $X$ is hemicompact.

(3) $C_{\tau_{p}}\left(X, \mathbb{E}^{1}\right)$ is metrizable if and only if $X$ is countable.

\section{5. $\alpha_{f}$-SPACES AND THE AsCOLI THEOREM}

Given a metric or a uniform space, $Y$, an Ascoli type theorem characterizes compactness in a function space $C(X, Y)$ in terms of equicontinuity plus natural conditions. For example, the basic Ascoli theorem deals with compactness in the function space $C([0,1])$ of all real-valued continuous functions on the unit interval endowed with the uniform topology. It states that a subset $F$ of $C([0,1])$ is compact if, and only if, $F$ is closed, bounded, and equicontinuous. Since the classical HeineBorel theorem states that a subset of $\mathbb{R}^{n}$ is compact if, and only if, it is closed and bounded, the basic Ascoli theorem can be viewed as fixing the problems of Heine-Borel theorem in $C([0,1])$. Recall that, in contrast to Heine-Borel theorem, in infinite-dimensional normed vector spaces, closed and bounded sets need not be compact and closed balls are never compact.

In the realm of function spaces of fuzzy-valued functions, we study Ascoli theorem in the spirit commented in the previous paragraph, that is, by means of equicontinuity plus extra conditions. The key idea is to analyze the relationship between $\alpha_{f^{-}}$-spaces and compactness in $C_{\tau_{\alpha}}\left(X, \mathbb{E}^{1}\right)$. As a fairly direct consequence of our results, we characterize when $C_{\tau_{u}}\left(X, \mathbb{E}^{1}\right)$ satisfies the fuzzy Ascoli theorem. Our approach follows the link between the exponential map and the Ascoli theorems as developed in [28].

First, some concepts are in order. Given a space $X$ and a metric space $(Y, d)$, a family $\left\{f_{i}\right\}_{i \in I} \subset F(X, Y)$ is called equicontinuous if for each $x \in X$ and each $\varepsilon>0$, there is a neighborhood $V$ of $x$ such that $d\left(f_{i}(y), f_{i}(x)\right)<\varepsilon$ for all $y \in V$ and all $i \in I$. In the case when $X$ is also a metric space, say $\left(X, d^{\prime}\right)$, the family $\left\{f_{i}\right\}_{i \in I} \subset F(X, Y)$ is said to be uniformly equicontinuous if for every $\varepsilon>0$, there is $\delta>0$ such that $d\left(f_{i}(y), f_{i}(x)\right)<\varepsilon$ for all $i \in I$ whenever $d^{\prime}(x, y)<\delta$. It is a well-known fact that, for a compact metric space $\left(X, d^{\prime}\right)$, equicontinuity is equivalent to uniform equicontinuity.

A subset $U \subset \mathbb{E}^{1}$ such that the families $\left\{u^{+}(-): u \in U\right\}$ and $\left\{u^{-}(-): u \in U\right\}$ are uniformly equicontinuous on $] 0,1]$ is named $] 0,1]$-uniformly equicontinuous.

The motivation for the results of this section is the following fuzzy version of the classical Ascoli theorem stated by Fang and Xue.

THEOREM 5.1. ([14, Theorem 4.2]) If $K$ is a compact metric space, then a closed subset $F$ of $C_{\tau_{u}}\left(K, \mathbb{E}^{1}\right)$ is compact if and only if the following conditions are satisfied:

(i) For each $k \in K$, the set $\{f(k): f \in F\}$ is $d_{\infty}$-bounded, that is, it is contained in a ball of center 0 in the metric space $\left(\mathbb{E}^{1}, d_{\infty}\right)$. 
(ii) $F$ is equicontinuous on $K$.

(iii) For each $k \in K$, the set $\{f(k): f \in F\}$ is ]0,1]-uniformly equicontinuous, i.e., $F$ is pointwise ]0,1]-uniformly equicontinuous.

Unfortunately the above theorem in based on a wrong characterization ([14, Theorem 2.4]) of the compact subsets of $\mathbb{E}^{1}$, as pointed out in [15]. To provide a right characterization we need to introduce several concepts. Namely, given a function $f:[0,1] \rightarrow \mathbb{R}$, let $f\left(\lambda_{0}+\right)$ denote the limit of $f$ when $\lambda$ approaches $\lambda_{0}$ from above (right).

DEFINITION 5.2. Let $\left\{f_{i}\right\}_{i \in I}$ be a family of functions defined from the unit interval $[0,1]$ into the reals. Given $\lambda_{0} \in\left[0,1\left[\right.\right.$ such that $f_{i}\left(\lambda_{0}+\right)$ exists for all $i \in I$, the family $\left\{f_{i}\right\}_{i \in I}$ is said to be almost-right-equicontinuous at $\lambda_{0}$ if, for every $\varepsilon>0$, there is $\delta>0$ such that $\left|f_{i}(\lambda)-f_{i}\left(\lambda_{0}+\right)\right|<\varepsilon$ for all $i \in I$ whenever $\left.\lambda \in\right] \lambda_{0}, \lambda_{0}+\delta[$.

Such a family of real-valued functions is said to be left-equicontinuous at a point $\left.\left.\lambda_{0} \in\right] 0,1\right]$ if, for all $\varepsilon>0$ and for all $i \in I$, there is $\delta>0$ such that $\left|f_{i}(\lambda)-f_{i}\left(\lambda_{0}\right)\right|<\varepsilon$ whenever $\left.\lambda \in] \lambda_{0}-\delta, \lambda_{0}\right]$. The family $\left\{f_{i}\right\}_{i \in I}$ is called left-equicontinuous (resp. almost-right-equicontinuous) if it is left-equicontinuous (resp. almost-right-equicontinuous) at every point of $] 0,1]$ (respectively, at every point of $[0,1[$ ).

We say that a subset $U \subset \mathbb{E}^{1}$ is both-sided equicontinuous if both $\left\{u^{+}(-): u \in U\right\}$ and $\left\{u^{-}(-): u \in U\right\}$ are almost-right-equicontinuous and left-equicontinuous.

DEFINITION 5.3. A subset $\mathscr{F}$ of $F\left(X, \mathbb{E}^{1}\right)$ is said to be pointwise both-sided equicontinuous if, for all $x \in X,\{f(x): f \in \mathscr{F}\}$ is both-sided equicontinuous.

DEFINITION 5.4. A subset $\mathscr{F}$ of $F\left(X, \mathbb{E}^{1}\right)$ is said to be pointwise $d_{\infty}$-bounded if, for all $x \in X,\{f(x): f \in \mathscr{F}\}$ is $d_{\infty}$-bounded in $\mathbb{E}^{1}$.

If $\tau$ is a topology on $C\left(X, \mathbb{E}^{1}\right)$, then $\left(X, \mathbb{E}^{1}, \tau\right)$ is said to satisfy the weak fuzzy Ascoli theorem if each $\tau$-closed, pointwise $d_{\infty}$-bounded, equicontinuous, pointwise both-sided equicontinuous subset of $C\left(X, \mathbb{E}^{1}\right)$ is $\tau$-compact. If also each $\tau$-compact subset is $\tau$-closed, pointwise $d_{\infty}$-bounded, equicontinuous, and pointwise both-sided equicontinuous, then $\left(X, \mathbb{E}^{1}, \tau\right)$ is said to satisfy the fuzzy Ascoli theorem.

The aim of this section is to establish fuzzy versions of the (weak) Ascoli theorem for topologies of the uniform convergence on the members of a cover of a space $X$, but first we need several previous results.

PROPOSITION 5.5. If $U \subset \mathbb{E}^{1}$ is both-sided equicontinuous, then so is cl $\mathbb{E}_{\mathbb{E}^{1}} U$.

Proof. Fix $\left.\left.\lambda_{0} \in\right] 0,1\right]$. By hypothesis, given $\varepsilon>0$, there is $\delta>0$ such that, for all $u \in U,\left|u^{+}(\lambda)-u^{+}\left(\lambda_{0}\right)\right|<\varepsilon / 3$ whenever $\left.\left.\lambda \in\right] \lambda_{0}-\delta, \lambda_{0}\right]$. Now, if $v \in \operatorname{cl}_{\mathbb{E}^{1}} U$, we can choose $u \in U$ with

$$
\sup _{\lambda \in] 0,1]}\left|v^{+}(\lambda)-u^{+}(\lambda)\right|<\varepsilon
$$

Then, if $\left.\lambda \in] \lambda_{0}-\delta, \lambda_{0}\right]$, we have

$$
\begin{aligned}
\left|v^{+}(\lambda)-v^{+}\left(\lambda_{0}\right)\right| \leq & \left|v^{+}(\lambda)-u^{+}(\lambda)\right| \\
& +\left|u^{+}(\lambda)-u^{+}\left(\lambda_{0}\right)\right|+\left|u^{+}\left(\lambda_{0}\right)-v^{+}\left(\lambda_{0}\right)\right|<7 \varepsilon / 3 .
\end{aligned}
$$


Thus, $\left\{v^{+}(-): v \in \mathrm{cl}_{\mathbb{E}^{1}} U\right\}$ is left-equicontinuous at each $\left.\left.\lambda_{0} \in\right] 0,1\right]$. Similar arguments show that the family $\left\{v^{-}(-): v \in \mathrm{cl}_{\mathbb{E}^{1}} U\right\}$ is also left-equicontinuous at each $\left.\left.\lambda_{0} \in\right] 0,1\right]$ and that both families are almost-right-equicontinuous. This completes the proof.

THEOREM 5.6. A subset $F \subseteq\left(\mathbb{E}^{1}, d_{\infty}\right)$ is precompact if and only if the following two conditions are satisfied:

(i) $F$ is $d_{\infty}$-bounded, and

(ii) $F$ is both-sided equicontinuous.

Proof. Suppose that $F$ is precompact. Since $\left(\mathbb{E}^{1}, d_{\infty}\right)$ is complete, $\operatorname{cl}_{\mathbb{E}^{1}} F$ is compact.

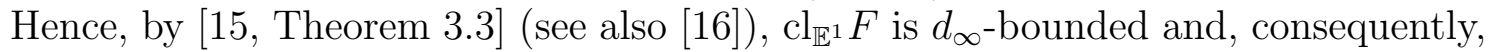
so is $F$. Similarly, by [15, Theorem 3.3], condition (ii) is satisfied.

To see the converse notice that, being $F d_{\infty}$-bounded, then so is $\operatorname{cl}_{\mathbb{E}^{1}} F$. In addition, by Proposition 5.5, $\mathrm{cl}_{\mathbb{E}^{1}} F$ is both-sided equicontinuous. Now the result follows from [15, Theorem 3.3].

THEOREM 5.7. For any space $X$, the $\tau_{p}$-closure of a subset $\mathscr{F}$ of $\left(\mathbb{E}^{1}\right)^{X}$ is a compact set if and only if the following two conditions are satisfied:

(1) $\mathscr{F}$ is pointwise $d_{\infty}$-bounded, and

(2) $\mathscr{F}$ is pointwise both-sided equicontinuous.

Proof. If $\mathscr{F}$ satisfies conditions (1) and (2), then so does $\mathrm{cl}_{\tau_{p}} \mathscr{F}$; indeed, condition (1) is straightforward and condition (2) follows from Proposition 5.5. Hence we can assume that $\mathscr{F}$ is $\tau_{p}$-closed. Consider now $\mathscr{F}$ as a subset of

$$
\prod_{x \in X} \operatorname{cl}_{\mathbb{E}^{1}}\{f(x): f \in \mathscr{F}\} \subset\left(\mathbb{E}^{1}\right)^{X} .
$$

Then $\mathscr{F}$ is a $\tau_{p^{-}}$closed subset of $\prod_{x \in X} \operatorname{cl}_{\mathbb{E}^{1}}\{f(x): f \in \mathscr{F}\}$ which is $\tau_{p^{-}}$-compact since each fiber is compact by Theorem 5.6. Thus, $\mathscr{F}$ is $\tau_{p}$-compact.

If $\mathscr{F} \subset\left(\mathbb{E}^{1}\right)^{X}$ is $\tau_{p}$-compact, then, since the projection map $\pi_{x}: \mathscr{F} \rightarrow \mathbb{E}^{1}$ defined, for all $x \in X$, as $\pi_{x}(f)=f(x)$, is continuous, we infer that $\{f(x): f \in \mathscr{F}\}$ is a compact subset of $\mathbb{E}^{1}$. Now, in order to obtain the desired result, it suffices to apply Theorem 5.6 for each $x \in X$.

COROLLARY 5.8. For any space $X$ and any cover $\alpha \subseteq \beta$, the $\tau_{\alpha}$-closure of a subset $\mathscr{F}$ of $C_{\tau_{\alpha}}\left(X, \mathbb{E}^{1}\right)$ is a compact set if and only if the two conditions in Theorem 5.7 are satisfied.

Given three topological spaces $X, Y, Z$, the map $\mu: F(X \times Y, Z) \rightarrow F(X, F(Y, Z))$ defined by the relation $\mu f(x)(y)=f(x, y)$ is called the exponential map. The restriction of this map to subspaces will also be denoted by $\mu$. The following results will be useful in the sequel. If $\alpha$ is a cover of $Y$ and $k$ is the cover of the compact subsets of $X$, then $k \times \alpha$ denotes the cover of $X \times Y$,

$$
k \times \alpha=\{A \times B: A \in k \text { and } B \in \alpha\} .
$$

THEOREM 5.9. [31, Proposition 2.2] If $K$ is a compact space and $Y$ is an $\alpha_{f}$-space, then $K \times Y$ is a $(k \times \alpha)_{f}$-space. 
THEOREM 5.10. Let $K$ be a compact space. If $Y$ is an $\alpha_{f}$-space $(\alpha \subseteq \beta)$, then

$$
\mu^{-1}\left(C\left(K, C_{\alpha}\left(Y, \mathbb{E}^{1}\right)\right) \subseteq C\left(K \times Y, \mathbb{E}^{1}\right)\right.
$$

Proof. By Theorem 5.9, it suffices to show that if $f \in \mu^{-1}\left(C\left(K, C_{\alpha}\left(Y, \mathbb{E}^{1}\right)\right)\right.$, then $\left.f\right|_{K \times A}$ has a continuous extension to $K \times Y$ for all $A \in \alpha$. For this, let $g \in$ $C\left(K, C_{\alpha}\left(Y, \mathbb{E}^{1}\right)\right)$. Being $\mathbb{E}^{1}$ a complete metric space, there exists, for all $x \in K$, a continuous extension $g(x)^{\mu}$ of $g(x)$ to the Dieudonné completion $\gamma Y$ of $Y$.

Now, for each $A \in \alpha$, consider the function $g_{A}$ from $K$ to $C_{\tau_{u}}\left(\operatorname{cl}_{\mu Y} A, \mathbb{E}^{1}\right)$ defined by the rule

$$
g_{A}(x)=\left.g(x)^{\mu}\right|_{\mathrm{cl}_{Y} A}, \quad x \in K .
$$

Since $g$ is continuous, it is an easy matter to show that so is $g_{A}$ and, consequently, $\mu^{-1}\left(g_{A}\right) \in C\left(K \times \operatorname{cl}_{Y} A\right)$ for all $A \in \alpha$. Being $K \times \operatorname{cl}_{Y} A$ compact, $\mu^{-1}\left(g_{A}\right)$ has a continuous extension to $K \times \gamma Y$ for all $A \in \alpha$. The result now follows from the fact that $\mu^{-1}\left(g_{A}\right)$ and $\mu^{-1}(g)$ coincide when restricted to $K \times A$.

THEOREM 5.11. Let $K$ be a compact space. If $X$ is an $\alpha_{f}$-space $(\alpha \subseteq \beta)$, then the equality $\mu\left(C\left(K \times X, \mathbb{E}^{1}\right)\right)=C\left(K, C_{\tau_{\alpha}}\left(X, \mathbb{E}^{1}\right)\right)$ holds.

Proof. A particular case of [34, Theorem 3.2] implies that, if $X$ is a $b_{f}$-space, then the equality $\mu\left(C\left(K \times X, \mathbb{E}^{1}\right)\right)=C\left(K, C_{\tau_{\beta}}\left(X, \mathbb{E}^{1}\right)\right)$ holds.

Next, the topology $\tau_{\alpha}$ being coarser than the topology $\tau_{\beta}$, a consequence of the above paragraph is the inclusion $\mu\left(C\left(K \times X, \mathbb{E}^{1}\right)\right) \subseteq C\left(K, C_{\tau_{\alpha}}\left(X, \mathbb{E}^{1}\right)\right)$.

Finally, the inclusion in Theorem 5.10 completes the proof.

We are now ready to characterize those $\left(X, \mathbb{E}^{1}, \tau_{\alpha}\right)$ satisfying the fuzzy weak Ascoli theorem. Let us recall that if $\alpha$ is a cover of $X$, then we have $\tau_{\alpha} \geq \tau_{p}$.

THEOREM 5.12. Let $X$ be a topological space. If $\alpha$ is a cover of $X$, then the following are equivalent:

(1) $\alpha \subseteq \beta$.

(2) For each (infinite) compact space $K$, the following inclusion holds:

$$
\mu\left(C\left(K \times X, \mathbb{E}^{1}\right)\right) \subseteq C\left(K, C_{\tau_{\alpha}}\left(X, \mathbb{E}^{1}\right)\right) .
$$

(3) $\left(X, \mathbb{E}^{1}, \tau_{\alpha}\right)$ satisfies the weak fuzzy Ascoli theorem.

Proof. (1) $\Longrightarrow(2)$ Let $K$ be a compact space and consider a continuous fuzzy-numbervalued function $f$ on $K \times X$. Since $\mathbb{E}^{1}$ is a complete metric space, $f$ admits a continuous extension $f^{\gamma}$ to $\gamma(K \times X)=K \times \gamma X$ (see [26, Theorem 5.1]). For each $B \in \alpha, \mathrm{cl}_{\gamma(X)} B$ is compact and by Theorem 5.11 ,

$$
\mu\left(\left.f^{\gamma}\right|_{K \times \mathrm{cl}_{\gamma(X)} B}\right) \in C\left(K, C_{\tau_{u}}\left(\operatorname{cl}_{\gamma(X)} B, \mathbb{E}^{1}\right)\right)
$$

which yields $\mu(f) \in C\left(K, C_{\tau_{\alpha}}\left(X, \mathbb{E}^{1}\right)\right)$. Thus, (2) holds.

$(2) \Longrightarrow(3)$ Let $K$ be a $\tau_{\alpha}$-closed, $d_{\infty}$-bounded, equicontinuous, pointwise bothsided equicontinuous subset of $C_{\tau_{\alpha}}\left(X, \mathbb{E}^{1}\right)$. By Theorem 5.7, $\mathrm{cl}_{\tau_{p}} K$ is compact. Moreover, by [24, Theorem 7.14], it is also equicontinuous. Now, by [24, Theorem 7.15], the evaluation mapping 


$$
\begin{aligned}
e: \operatorname{cl}_{\tau_{p}} K \times X & \rightarrow \mathbb{E}^{1} \\
(f, x) & \rightarrow f(x)
\end{aligned}
$$

is a continuous function. By condition (2), the function $\mu(e): \mathrm{cl}_{\tau_{p}} K \rightarrow C_{\tau_{\alpha}}\left(X, \mathbb{E}^{1}\right)$ is continuous.

Notice that, since $e$ is the evaluation mapping, $\mu(e)$ is the inclusion mapping. Thus, $\mathrm{cl}_{\tau_{p}} K$ is $\tau_{\alpha}$-compact. Taking into account that $K$ is a $\tau_{\alpha}$-closed subset of $\mathrm{cl}_{\tau_{p}} K$, we have that $K$ is $\tau_{\alpha}$-compact.

$(3) \Longrightarrow(1)$ Suppose there is $B \in \alpha$ which is not bounded. Hence we can find a locally finite sequence $\left\{U_{n}: n \in \mathbb{N}\right\}$ of pairwise disjoint open sets meeting $B$ which is not finite. Now choose a sequence $\left\{v_{n}: n \in \mathbb{N}\right\}$ of elements of $\mathbb{E}^{1}$ such that $d_{\infty}\left(0, v_{n}\right)>1$ for all $n \in \mathbb{N}$. For each $n \in \mathbb{N}$, fix $x_{n} \in U_{n} \cap B$. By Proposition 3.5 of [23] there is a function $f_{n} \in C\left(X, \mathbb{E}^{1}\right)$ satisfying $f_{n}\left(x_{n}\right)=v_{n}$ and $\left.f_{n}\right|_{X \backslash U_{n}}=0$. Next, for each $n \in \mathbb{N}$, consider the function $g_{n}$ on $X$ defined as

$$
g_{n}(x)=\sum_{k=1}^{n} f_{k}(x) \quad \text { for all } x \in X .
$$

According to [23, Proposition 3.1], $g_{n}$ is continuous for all $n \in \mathbb{N}$. It is easy to see that the sequence $\mathscr{B}=\left\{g_{n}: n \in \mathbb{N}\right\}$ converges in $C_{\tau_{p}}\left(X, \mathbb{E}^{1}\right)$ which implies that it is a $\tau_{p}$-relatively compact subset. Being $\tau_{\alpha}$ stronger than $\tau_{p}$, the $\tau_{\alpha}$-closure of $\mathscr{B}$ is contained in its $\tau_{p}$-closure. Thus, by Theorem 5.7 , the $\tau_{\alpha}$-closure of $\mathscr{B}$ is pointwise $d_{\infty}$-bounded, and pointwise both-sided equicontinuous. We shall prove that $\mathscr{B}$ is equicontinuous. For this, fix $x \in X$. We shall consider two cases:

Case 1. $x \notin \bigcup_{n \in \mathbb{N}} U_{n}$.

Being the sequence $\left\{U_{n}: n \in \mathbb{N}\right\}$ locally finite, we can choose a nonempty open set $V$ with $x \in V$ such that $V \cap \bigcup_{n \in \mathbb{N}} U_{n}=\emptyset$; this implies that

$$
d_{\infty}\left(g_{n}(y), g_{n}(x)\right)=0
$$

for all $y \in V$ and all $n \in \mathbb{N}$.

Case 2. There is $n \in \mathbb{N}$ such that $x \in U_{n}$.

Let $\varepsilon>0$. Consider the open set $V=U_{n} \cap f_{n}^{-1}\left(B_{\varepsilon}\left(f_{n}(x)\right)\right.$. Then, for all $y \in V$, we have

$$
d_{\infty}\left(g_{k}(y), g_{k}(x)\right)<\varepsilon
$$

for all $k \geq n$.

Case 1 , together with Case 2 , shows that $\mathscr{B}$ is equicontinuous at $x$. If $x$ is an arbitrary point of $X$, then the family $\mathscr{B}$ is equicontinuous on $X$. Since $\tau_{\alpha} \geq \tau_{p}$, Theorem 7.14 of [24] tells us that the $\tau_{\alpha}$-closure of $\mathscr{B}$ is equicontinuous as well. We conclude the proof by proving that $\mathscr{B}$ is not relatively compact in $C_{\tau_{\alpha}}\left(X, \mathbb{E}^{1}\right)$. Indeed, note that $g_{s}\left(x_{j}\right)=0$ and $g_{k}\left(x_{j}\right)=v_{j}$ whenever $s<j<k$ and, consequently, by choice of the sequence $\left\{v_{n}: n \in \mathbb{N}\right\}$, we have $d_{\infty}\left(g_{s}\left(x_{j}\right), g_{k}\left(x_{j}\right)\right)>1$ whenever $s<j<k$. Thus, $\mathscr{B}$ is not relatively compact in $C_{\tau_{\alpha}}\left(X, \mathbb{E}^{1}\right)$. 
It is convenient to provide an example of a function space satisfying the weak fuzzy Ascoli theorem but not the fuzzy Ascoli theorem to illustrate the notions that are being discussed. Recall that a function $f: X \times Y \rightarrow Z$ is said to be separately continuous if $\left.f\right|_{X \times\{y\}}$ and $\left.f\right|_{\{x\} \times Y}$ are continuous for all $x \in X$ and all $y \in Y$. It is worth mentioning that any real-valued continuous function defined on the product $X \times Y$ of two pseudocompact spaces can be extended to a separately continuous (not necessarily continuous) function defined on $\beta X \times \beta Y$ where, as usual, $\beta M$ stands for the Stone-Čech compactification of a space $M$.

EXAMPLE 5.13. Let $K \times X$ be a product space with $K$ compact. Assume that there is a separately continuous (but not continuous) fuzzy-number-valued function $f$ on $K \times X$. By Theorem $5.12, C_{\tau_{p}}\left(X, \mathbb{E}^{1}\right)$ satisfies the weak fuzzy Ascoli theorem. We shall prove that $C_{\tau_{p}}\left(X, \mathbb{E}^{1}\right)$ does not satisfy the fuzzy Ascoli theorem. For this, we claim that $\mu(f) \in C\left(K, C_{\tau_{p}}\left(X, \mathbb{E}^{1}\right)\right)$. Indeed, the function $f$ being continuous on $\{k\} \times X$ for all $k \in K$, the function $\mu(f)(k) \in C\left(X, \mathbb{E}^{1}\right)(k \in K)$. Now, to see that $\mu(f)$ is continuous at every point $k \in K$, consider a net $k_{\delta}$ converging to $k \in K$. Since $\left.f\right|_{K \times\{x\}}$ is continuous for all $x \in X$, we have that $f\left(k_{\delta}, x\right)$ converges to $f(k, x)$ for all $x \in X$. Thus, $\mu(f)$ is continuous.

Suppose now, to derive a contradiction, that $C_{\tau_{p}}\left(X, \mathbb{E}^{1}\right)$ satisfies the fuzzy Ascoli theorem. Then the compact set $\mu(f)(K)$ is equicontinuous. By [24, Theorem 7.15], the evaluation function

$$
\begin{aligned}
e: \mu(f)(K) \times X & \rightarrow \mathbb{E}^{1} \\
(h, x) & \rightarrow h(x)
\end{aligned}
$$

$(h \in \mu(f)(K), x \in X)$ is continuous. So $f=e \circ\left(\mu(f) \times \mathrm{id}_{X}\right)$ is also continuous, a contradiction. Therefore $C_{\tau_{p}}\left(X, \mathbb{E}^{1}\right)$ does not satisfy the fuzzy Ascoli theorem.

THEOREM 5.14. Let $\alpha \subseteq \beta$ be a cover of a space $X$. If $X$ is an $\alpha_{f}$-space, then $\left(X, \mathbb{E}^{1}, \tau_{\alpha}\right)$ satisfies the fuzzy Ascoli theorem.

Proof. By Theorem 5.12, we only need to prove that a compact subset $K$ of $C_{\tau_{\alpha}}\left(X, \mathbb{E}^{1}\right)$ is $\tau_{\alpha}$-closed, pointwise $d_{\infty}$-bounded, pointwise both-sided equicontinuous and equicontinuous. Consider the (continuous) inclusion mapping $i$ from $K$ into $C_{\tau_{\alpha}}\left(X, \mathbb{E}^{1}\right)$. By Theorem 5.11, the evaluation mapping $\mu^{-1}(i)$ is continuous. Since $\tau_{\alpha} \geq \tau_{p}$, equicontinuity of $K$ follows from [24, Theorem 7.19 and Theorem 7.20]. Now, being $K$ $\tau_{p}$-compact, $K$ is pointwise $d_{\infty}$-bounded and pointwise both-sided equicontinuous. This completes the proof thanks to Theorem 5.6.

COROLLARY 5.15. The following statements hold:

(1) If $X$ is a $b_{f}$-space, then $\left(X, \mathbb{E}^{1}, \tau_{\beta}\right)$ satisfies the fuzzy Ascoli theorem.

(2) If $X$ is a $k_{r}$-space, then $\left(X, \mathbb{E}^{1}, \tau_{c o}\right)$ satisfies the fuzzy Ascoli theorem.

The previous corollary has several applications. Two of them are stated in what follows. Although an arbitrary product of pseudocompact groups is a pseudocompact group (see [10]), it is a trivial fact that an arbitrary product of locally pseudocompact groups fails to be locally pseudocompact. However, an arbitrary product of locally pseudocompact groups is a $b_{f}$-group, that is, a topological group whose underlying space is a $b_{f}$-space (see [34, Theorem 4.3]). Thus, we have 
COROLLARY 5.16. If $G$ is an arbitrary product of locally pseudocompact groups, then $\left(G, \mathbb{E}^{1}, \tau_{\beta}\right)$ satisfies the fuzzy Ascoli theorem.

The product of two $k_{r}$-spaces need not be a $k_{r}$-space ([22]) but there are several interesting cases where an arbitrary product of $k_{r}$-spaces is a $k_{r}$-space as well. For example, if $X_{\alpha}$ is a $k_{r}$-space pseudocompact for all $\alpha \in I$, then so is $X=\prod_{\alpha \in I} X_{\alpha}$ ([29, Theorem 4.2]). This fact allows us to state the following

COROLLARY 5.17. If $X$ is an arbitrary product of $k_{r}$-pseudocompact spaces, then $\left(X, \mathbb{E}^{1}, \tau_{c o}\right)$ satisfies the fuzzy Ascoli theorem.

For the important case of the uniform convergence, we have

COROLLARY 5.18. For a space $X$, the following conditions are equivalent:

(1) $X$ is pseudocompact.

(2) $\left(X, \mathbb{E}^{1}, \tau_{u}\right)$ satisfies the weak fuzzy Ascoli theorem.

(3) $\left(X, \mathbb{E}^{1}, \tau_{u}\right)$ satisfies the fuzzy Ascoli theorem.

Proof. By Theorem 5.12, it is apparent that (1) and (2) are equivalent and that $(3) \Longrightarrow(1)$. Finally $(1) \Longrightarrow(3)$ follows from Theorem 5.14 .

COROLLARY 5.19. [14, Theorem 4.2] For each compact metric space $K,\left(K, \mathbb{E}^{1}, \tau_{u}\right)$ satisfies the fuzzy Ascoli theorem.

REMARK 5.20. We would like to thank the referee for drawing our attention to a recent interesting paper by $\mathrm{H}$. Huang ([21]). In his manuscript, the author provides a complete study of the endograph metric, $H_{\text {end }}$, on general fuzzy sets (containing fuzzy numbers) which, combined with the ideas presented in this paper, opens new perspectives for future research on the realm of fuzzy-number-valued functions. More precisely, Huang's results on compactness and our techniques could be applied to obtain versions of (weakly) Arzelà-Ascoli theorem for the space $C\left(X,\left(\mathbb{E}^{1}, H_{\text {end }}\right)\right)$. It is worth noting that, since $H_{\text {end }}(u, v) \leq d_{\infty}(u, v)$ for all $u, v \in \mathbb{E}^{1}([8])$, the identity map is continuous from $C_{\tau_{\alpha}}\left(X,\left(\mathbb{E}^{1}, d_{\infty}\right)\right)$ into $C_{\tau_{\alpha}}\left(X,\left(\mathbb{E}^{1}, H_{\text {end }}\right)\right)(\alpha$ a bornology on $X)$. Thus, every compact subset of $C_{\tau_{\alpha}}\left(X,\left(\mathbb{E}^{1}, d_{\infty}\right)\right)$ is compact in $C_{\tau_{\alpha}}\left(X,\left(\mathbb{E}^{1}, H_{\text {end }}\right)\right)$.

\section{Conclusion}

The aim of this paper is to describe three topological properties, namely, completeness, metrizability and compactness, in the space of continuous (with respect to the supremum metric) fuzzy-number-valued functions defined on a topological space $X$. We conclude that, when we endow these function spaces with the (commonly used) topology of uniform convergence on the members of a cover of $X$, such properties are closely related to those covers contained in the cover of all bounded sets. Completeness and metrizability had not been addressed in the fuzzy literature yet, while our results on compactness (that is, Ascoli type results) correct and improve some previously published ones. We expect this research to be useful for future developments in Fuzzy Analysis. 


\section{REFERENCES}

[1] R.A. Alò and L.I. Sennett, Extending Linear Space-Valued Functions, Math. Ann. 191 (1971), 79-86.

[2] A.V. Arkhangels'kii, Function spaces in the topology of pointwise convergence and compact sets, Russian Math. Surveys 39 (1984), 9-56.

[3] A.V. Arkhangels'kii, When the Dieudonné completion of a topological group is a paracompact p-space, Topology Proc. 25 (2000), 1-15.

[4] C. Arzelà, Sull' integrabilita della equazioni differenziali ordinarie, Mem. Accad. Bologna 5 (1895-1896) no. 5, 257-270.

[5] G. Ascoli, Le curve limite di una varietà data di curve, Mem. Accad. Lincei 18 (1883) no. 3, $521-586$

[6] B. Bede and S. Gal, Generalizations of the differentiability of fuzzy-number-valued functions with applications to fuzzy differential equations, Fuzzy Sets and Systems 151 (2005) 581-599.

[7] J.L. Blasco and M. Sanchis, On the product of two $b_{f}$-spaces, Acta Math. Hungar. 62 (1993) no. $1-2,111-118$.

[8] J. P. Boronski and J. Kupka, The topology and dynamics of the hyperspaces of normal fuzzy sets and their inverse limit spaces, Fuzzy Sets and Systems 321 (2017), 90-100.

[9] H. Buchwalter, Produit topologique, produit tensoriel et c-repletion, Actes du Colloque d'Analyse Fonctionnelle de Bordeaux (Univ. Bordeaux, 1971), pp. 51-71. Bull. Soc. Math. France, Mem. No. 31-32, Soc. Math. France, Paris, 1972 (in French).

[10] W.W. Comfort and K.A. Ross, Pseudocompactness and uniform continuity in topological groups, Pacific J. Math. 16 (1966), 483-496.

[11] W. Cong-Xin and M. Ming, Embedding problem of fuzzy number space: Part I, Fuzzy Sets and Systems 44 (1991), 33-38.

[12] P. Diamond and P. Kloeden, Metric spaces of fuzzy sets. Theory and applications. World Scientific Publishing Co., Inc., River Edge, NJ, 1994.

[13] R. Engelking, General Topology, 2nd Ed., Sigma Series in Pure Mathematics, vol. 6, Heldermann Verlag, Berlin, 1989.

[14] J-X. Fang and Q-Y. Xue, Some properties of the space of fuzzy-valued continuous functions on a compact set, Fuzzy Sets and Systems 11 (2009), 1620-1631.

[15] J.J. Font and M. Sanchis, Sequentially compact subsets and monotone functions: An application to fuzzy theory, Topology Appl. 192 (2015), 113-122.

[16] J.J. Font and M. Sanchis, Compact subsets in the fuzzy number space, Proc. ISWAT'14, 63-75.

[17] T.E. Gantner, Extensions of uniformly continuous pseudometrics, Trans. Amer. Math. Soc. 132 (1968), 147-157.

[18] L. Gillman and M. Jerison, Rings of continuous functions. Reprint of the 1960 edition. Graduate Texts in Mathematics, No. 43. Springer-Verlag, New York-Heidelberg, 1976.

[19] R. Goetschel and W. Voxman, Elementary fuzzy calculus, Fuzzy Sets and Systems 18 (1986), $31-42$.

[20] S. Hai, Z. Gong and H. Li, Generalized differentiability for n-dimensional fuzzy-number-valued functions and fuzzy optimization, Inform. Sci. 374 (2016) 151-163.

[21] H. Huang, Characterizations of endograph metric and $\Gamma$-convergence on fuzzy sets, Fuzzy Sets and Systems. Available online 6 March 2018 (DOI 10.1016/j.fss.2018.02.014).

[22] M. Hušek, Products of quotients and of $k^{\prime}$-spaces, Comment. Math. Univ. Carolinae 12 (1971), $61-68$.

[23] D.R. Jardón and M. Sanchis, Pointwise convergence topology and function spaces in fuzzy analysis, Iranian J. Fuzzy Syst. 15 (2018), 1-21.

[24] J.L. Kelley, General Topology, New York, D. van Nostrand Company Inc., 1955.

[25] B.S. Lee and S.J. Cho, A fixed point theorem for contractive-type fuzzy mappings, Fuzzy Sets and Systems 61 (1994) no. 3, 309-312.

[26] K. Morita, Topological completions and M-spaces, Sci. Rep. Tokyo Kyoiku Daigaku Sect. A 10 (1970) 271-288. 
[27] N. Noble, A note on z-closed projections, Proc. Amer. Math. Soc. 23 1969, 73-76.

[28] N. Noble, Ascoli theorems and the exponential map, Trans. Amer. Math. Soc. 143 (1969), $393-411$.

[29] N. Noble, The continuity of functions on Cartesian products, Trans. Amer. Math. Soc. 149 (1970), 187-198.

[30] J.Y. Park and J.U. Jeong, Fixed point theorems for fuzzy mappings, Fuzzy Sets and Systems 87 (1997) no. 1, 111-116.

[31] R. Pupier, Topological completion of a product, Rev. Roumaine Math. Pures Appl. 19 (1974), 925-933.

[32] E.A. Reznichenko, Extension of functions defined on products of pseudocompact spaces and continuity of the inverse in pseudocompact groups. Topology Appl. 59 (1994), no. 3, 233-244.

[33] R. Rodríguez-López, Monotone method for fuzzy differential equations, Fuzzy Sets and Systems 159 (2008) 2047-2076.

[34] M. Sanchis, Continuous functions on locally pseudocompact groups, Topology Appl. 86 no. 1 (1998), 5-23.

[35] M. Sanchis, Problems on bounded subsets, Questions Answers Gen. Topology 28 (2010) no. 1, $65-79$.

[36] H.L. Shapiro, Extensions of pseudometrics, Canad. J. Math. 18 (1966), 981-998.

[37] C. Wu and Z. Gong, On Henstock integral of fuzzy-number-valued functions (I), Fuzzy Sets and Systems 120 (2001), 523-532.

[38] C.X. Wu and C. Wu, The supremum and infimum of the set of fuzzy numbers and its application, J. Math. Anal. Appl. 210 (1997) no. 2, 499-511.

[39] C.X. Wu and C. Wu, Some notes on the supremum and infimum of the set of fuzzy numbers, Fuzzy Sets and Systems 103 (1999) no. 1, 183-187.

[40] C. Wu and G. Wang, Convergence of sequences of fuzzy numbers and fixed point theorems for increasing fuzzy mappings and application, Fuzzy Sets and Systems 130 (2002) no. 3, 383-390.

[41] G.Q. Zhang, The convergence for a sequence of fuzzy integrals of fuzzy number-valued functions on the fuzzy set, Fuzzy Sets and Systems 59 (1993) no. 1, 43-57.

Current address: Institut Universitari de Matemàtiques i Aplicacions de Castelló (IMAC), Universitat Jaume I, Campus del Riu Sec. s/n, 12071 Castelló (Spain)

E-mail address: font@uji.es, dsanchis@uji.es, sanchis@uji.es 